\title{
Bistability in the perception of motion and stationarity: Effects of temporal asymmetry
}

\author{
KATHLEEN E. EASTMAN \\ Northern Arizona University, Flagstaff, Arizona \\ and \\ HOWARD S. HOCK \\ Florida Atlantic University, Boca Raton, Florida
}

\begin{abstract}
Evidence for bistability in the perception of motion and stationarity was obtained for a displaced dotfigure embedded in a background of randomly moving noise dots. In the temporal symmetry condition, the figure was presented for the same duration in its two locations; either figure motion or random motion was perceived, depending on the number of noise dots. In the temporal asymmetry condition, the figure was presented for different durations in its two locations; figure motion, a single, stationary figure in a fixed position, or random noise was perceived, again depending on the number of noise dots. Competition between these percepts was established by an analysis of switching rates and by an experiment demonstrating the presence of hysteresis as noise levels were gradually increased and decreased across the figure-motion and figure-stationarity boundaries. This evidence for bistability in the perception of figure motion and figure stationarity (one or the other, but not both, was perceived for the same stimulus) suggested the presence of strong inhibitory competition between motion- and position-detecting mechanisms.
\end{abstract}

It has recently been shown that perception can alternate between movement and stationarity without any change in the stimulus - that is, perception can be bistable at the 50\% threshold (Hock, Balz, \& Eastman, 1996; Hock, Kogan, \& Espinoza, 1997). When motion is perceived, it indicates that the stimulus is adequate for the activation of motion-sensitive detectors. When it fails for the same stimulus, inhibitory interactions, resulting in the suppression of motion perception, are implicated. This article is concerned with whether the failure to perceive motion for a seemingly adequate stimulus is the result of inhibitory interactions within the ensemble of stimulusactivated motion detectors (in effect, self-inhibition within the motion-detecting system) or whether it is the result of inhibition of motion detectors by concurrently activated position detectors.

In one example of motion/stationarity bistability, Hock et al. (1996) presented a long row of evenly spaced dots during a series of frames in such a way that the row of dots was displaced by half its interdot distance on successive

This research is based on K.E.E.'s doctoral dissertation. Part of this work was originally presented in May 1996 at the Association for Research in Vision and Ophthalmology annual conference in Fort Lauderdale, Florida. We thank three anonymous reviewers for their helpful comments and suggestions. Correspondence concerning this article should be addressed to K. E. Eastman, Department of Psychology, Northern Arizona University, Flagstaff, AZ 86011-5106 (e-mail: k.eastman@nau.edu).

-Accepted by previous editor, Myron L. Braunstein frames. When the interframe-interval (IFI) was $0 \mathrm{msec}$, motion was always perceived over a wide range of interdot distances. However, the introduction of a blank IFI of $60 \mathrm{msec}$ between successive frames eliminated motion perception for their smallest interdot distance $\left(0.22^{\circ}\right)$ and established motion/stationarity bistability for their next largest interdot distance $\left(0.33^{\circ}\right)$ - that is, for the latter, motion and stationarity were each perceived on about half the trials.

In a second example, Hock et al. (1997) simultaneously presented two small, visible squares near each other; the luminance of each was greater than the background luminance. On odd-numbered frames, the luminance was greater for the square on the left; on even-numbered frames it was greater for the square on the right (i.e., the luminance values were exchanged on successive frames). When the background-relative luminance contrast (BRLC) was relatively large, the brighter of the two squares was perceived as moving through the intervening space. When it was small, motion perception was lost. For intermediate BRLC values, motion and stationarity were perceived equally often; perception was bistable.

It was of particular significance in the above studies that the alternative percepts were temporally stable; either stable motion or stable stationarity was perceived, with relatively little switching between them and with no intermediate state of uncertainty regarding which was perceived. The existence of alternative, temporally stable percepts for the same stimulus is important, because it indicates that there are competitive interactions among the relevant detectors. If the stimulus is adequate for the 
occurrence of two percepts and only one is experienced, there must be strong inhibitory interactions that suppress the other. The present experiments address the nature of these inhibitory interactions.

The issue of competition between perceptual states (motion and stationarity) can be expressed in terms of relevant neurophysiological evidence. Orban, Kennedy, and Bullier (1986) have described cortical neurons in the monkey that are velocity selective. In addition to those neurons selectively tuned to different narrow bands of velocities (bandpass, motion detectors) and those responding to broad bands of velocity, neurons were found that responded selectively to stationary or very slowly moving stimuli (velocity lowpass, position detectors). Because of receptive field overlap, both bandpass (motion) and lowpass (position) detectors can be directly activated by the same, bistable apparent-motion stimulus. The failure to perceive motion in the above examples could thereby be due either to inhibitory interactions within the ensemble of stimulus-activated bandpass (motion) detectors or to inhibitory interactions between stimulus-activated bandpass (motion) and lowpass (position) detectors.

Distinguishing between these alternatives in the Hock et al. (1996) and Hock et al. (1997) studies was not possible, because the perception of position was irrelevant to subjects' reports of whether or not motion was perceived. The need for position perception was made explicit in the present study, which required that subjects perceive a displaced three-dot figure in a background of random dot motion. As in most experiments of this kind (e.g., Braddick, 1974), the figure can be segregated from the background on the basis of perceived figure motion. What is distinctive in this study is that a temporal manipulation has been discovered that also allows the figure to be segregated from the background on the basis of its perceived position. The existence of motion/stationarity bistability under these circumstances would provide evidence for inhibitory competition between the perception of motion and the perception of position for the same apparent-motion stimulus.

\section{EXPERIMENT 1}

For standard apparent-motion stimuli, a displaced figure is presented for the same duration at each of its two alternating locations. Under these conditions (temporal symmetry), the motion of the figure is perceived until the number of noise dots in which the figure is embedded is increased to a point at which figure motion is lost and only random motion is perceived. This failure to perceive the figure in the absence of figure motion is typical of studies in which displaced figures are composed of discrete elements and presented against a background of random noise composed of the same elements (e.g., Braddick, 1974; Casco \& Morgan, 1987; Casco, Morgan, $\&$ Ward, 1989). In this experiment and the experiments that follow, we have also studied temporally asymmetric apparent-motion stimuli. For these stimuli, the displaced figure is presented for different durations at each of its two alternating locations.

Preliminary observations indicated that something unique occurs with temporally asymmetric apparent motion. Figure motion is perceived when the number of noise dots is small; however, as the number of noise dots is increased, the perception of the figure motion is suddenly lost and replaced by the perception of a single, stationary figure, with no intervening state of perceptual uncertainty. Near this transitional noise level, perception appears to be bistable; either a moving figure or a stationary figure is perceived, but never at the same time. This phenomenon was investigated by presenting a figure composed of dots in two alternating locations, varying the number of noise dots and having subjects report whether they perceived the figure in motion, whether they perceived the figure regardless of whether or not it was moving, or whether they perceived only random dot motion.

\section{Method}

Stimuli. Viewing was binocular in a darkened room. A chinrest maintained a constant viewing distance of $40 \mathrm{~cm}$, from which a 1-pixel dot subtends approximately $3 \mathrm{~min}$ of arc on a Macintosh 13 -in RGB monitor with a frame rate of $67 \mathrm{~Hz}$. The three vertically aligned dots composing the figure (the interdot distance was 18 arc $\mathrm{min}$ ) and the randomly located noise dots were small white squares (size, $3 \times 3$ arc min; luminance, $32.5 \mathrm{~cd} / \mathrm{m}^{2}$ ). They were presented against a dark background (luminance, $0.01 \mathrm{~cd} / \mathrm{m}^{2}$ ).

The figure was presented at each of its horizontally separated locations during the two frames composing each display cycle. Every trial comprised four display cycles, during which the figure was displaced back and forth by either $18,30,42$, or 54 arc min on successive frames. The initial figure location (left vs. right) was randomly determined on each trial.

The two locations of the figure were symmetrical with respect to the center of a $2^{\circ} \times 2^{\circ}$ region (with no visible boundaries) in the center of the screen. There were 81 evenly spaced locations in this region that could be occupied by noise dots (none of these locations could be occupied by the dots composing the figure). On each trial, the 3-dot figure was presented along with $2,4,6,8,10,12,14,16$, 18 , or 20 noise dots. The location of each noise dot changed randomly from one frame to the next within each trial.

The temporal frequency for both the temporal symmetry and asymmetry conditions was $3.3 \mathrm{~Hz}$ (each display cycle had a duration of $300 \mathrm{msec}$ ). ${ }^{1}$ In the temporal symmetry condition, the duration of every frame was $150 \mathrm{msec}$; the equal durations resulted in a 0.50 duty cycle at each of the figure's locations. In the temporal asymmetry condition, the frame duration was $225 \mathrm{msec}$ for one frame in each display cycle and $75 \mathrm{msec}$ for the other; the unequal durations resulted in duty cycles of 0.75 at one location of the figure and 0.25 at the other. Whether the 225 - or the 75 - $\mathrm{msec}$ frame duration was associated with the left or the right location of the figure was randomly determined for each trial. In the absence of noise dots, horizontal motion of the figure was always perceived for both temporal conditions.

Design. There were three experimental variables: temporal condition (two levels: temporal symmetry or asymmetry), figure displacement (four levels: 18, 30, 42, or 54 min of arc), and the number of randomly located noise dots (ten levels: $2-20$ ). Their orthogonal combination produced $80(2 \times 4 \times 10)$ stimulus configurations, each of which was tested 40 times over the course of eight daily 45 min testing sessions. The four displacements were tested in separate 
blocks of 100 trials during each experimental session (the order of the blocks was Latin-Square balanced), and the 10 different noise levels were varied randomly within subblocks of 10 trials. The temporal symmetry and asymmetry conditions were tested on alternating days; half the subjects began with one, half with the other.

Procedure. The subjects made two responses at the end of each trial, the first by pressing one of two keys to indicate whether or not they saw the figure for the entire trial, and the second by pressing one of two keys to indicate whether or not they saw the horizontal motion of the figure during the entire trial.

Subjects. Four female Florida Atlantic University students (ages 20-41 years) with normal or corrected-to-normal vision volunteered to participate in this experiment and the experiments that follow. Three of the 4 subjects were naive as to the purpose of the experiment. The 4 th (K.E.) was one of the authors.

\section{Results}

The percentage of trials for which the subject responded yes was averaged for each noise level to obtain psychometric functions for the two response categories. The psychometric functions (averaged over the 4 subjects) for perceiving the figure (response one) and its motion (response two) were virtually identical in the temporal symmetry condition (Figure 1, left panels), indicating that for all the displacement distances, whenever subjects perceived the displaced three-dot figure in the noisy background, they perceived it as moving. When the perception of figure motion was lost, only random motion was perceived.

In contrast, the two psychometric functions were quite different in the temporal asymmetry condition (Figure 1, right panels). With only a few noise dots, the figure was perceived as being in motion. With more noise dots, the perception of motion was lost, but now a single, stationary figure was perceived (i.e., it was always in the same position). For still more noise dots, the perception of the figure was lost, and only random motion was perceived.

Probit analyses were performed separately for every subject in each daily session and for each of the four displacements. Thresholds of $50 \%$ were calculated for perceiving the figure in motion (i.e., the number of noise dots that resulted in figure motion being perceived on half the trials) and for perceiving the figure, regardless of whether or not it was in motion (i.e., the number of noise dots that resulted in the figure being perceived on half the trials). These results are shown in Figure 2. For all 4 subjects, figure motion was perceived for higher noise levels in the temporal symmetry condition than in the temporal asymmetry condition (i.e., the perception of figure motion tolerated more noise dots in the temporal symmetry condition).

In addition, there was a tendency for the perception of figure motion to decrease as the size of the figure displacement increased; fewer noise dots were sufficient to eliminate the perception of figure motion. This trend, which was consistent with previous studies (e.g., Braddick, 1974; Ramachandran \& Anstis, 1983), was observed for all 4 of the subjects in the temporal symmetry condition and for 2 of the subjects in the temporal asymmetry condition (for the latter, floor effects may have constrained the effect of displacement distance). Similar trends were observed for the perception of a single, stationary figure in the temporal asymmetry condition, possibly owing to effects of spatial attention; the likelihood of perceiving the figure might be larger when its two possible locations are close enough to fall within a single attentional spotlight (Posner, 1980).

Possible effects of figure masking on motion perception. An additional experiment was conducted in order to show that the perception of a single, stationary figure in the temporal asymmetry condition was not due to the random noise dots masking the perception of the figure during the frames in which it was presented for only $75 \mathrm{msec}$. In the additional experiment, the perception of figure motion in a temporal symmetry condition with alternating $75-\mathrm{msec}$ durations (displacement size = $30 \mathrm{arc} \mathrm{min)}$ tolerated a relatively large number of noise dots $(50 \%$ threshold $=7.8$ noise dots for Subject K.E.), as compared with a temporal asymmetry condition with alternating 225- and 75-msec frames, as in Experiment 1 ( $50 \%$ threshold $=3.8$ noise dots for Subject K.E. retested). If the loss of motion in the temporal asymmetry condition in Experiment 1 was due to masking of the figure by noise dots during the $75-\mathrm{msec}$ frames, figure motion would have been eliminated by a similar number of noise dots when the duration of both frames was $75 \mathrm{msec}$.

Rather than masking affecting the perception of figure motion by "hiding" the figure during the 75 -msec frames, figure motion minimizes the effects of noise masking on the perception of the figure. That is, when the motion signal generated by the displaced figure is sufficiently strong, it helps with the segregation of the figure from the noise. This was the case in the temporal symmetry condition (for both the 150- and the 75-msec frame durations), but was less so in the temporal asymmetry condition.

Attentional feature-tracking mechanisms. Attentional feature-tracking mechanisms (Cavanagh, 1992) can likewise be ruled out as the basis for motion perception in this paradigm. There is no reason why feature tracking would be more difficult (i.e., more susceptible to noise) when the frame duration alternates between 225 and $75 \mathrm{msec}$, as compared with when the frame duration is always $75 \mathrm{msec}$.

Perception of the stationary figure. The perception of the stationary figure in the temporal asymmetry condition was specifically due to the asymmetry in frame durations, rather than to the particular durations tested ( $225 \mathrm{msec}$ alternating with $75 \mathrm{msec}$ ). In the additional experiment described above, a stationary figure was never perceived when the duration of every frame was $75 \mathrm{msec}$. Nor was it perceived when the duration of every frame was $225 \mathrm{msec}$ (or $150 \mathrm{msec}$ in the temporal symmetry condition of the primary experiment). Regardless of frame duration, when the figure was perceived under conditions 
TEMPORAL SYMMETRY
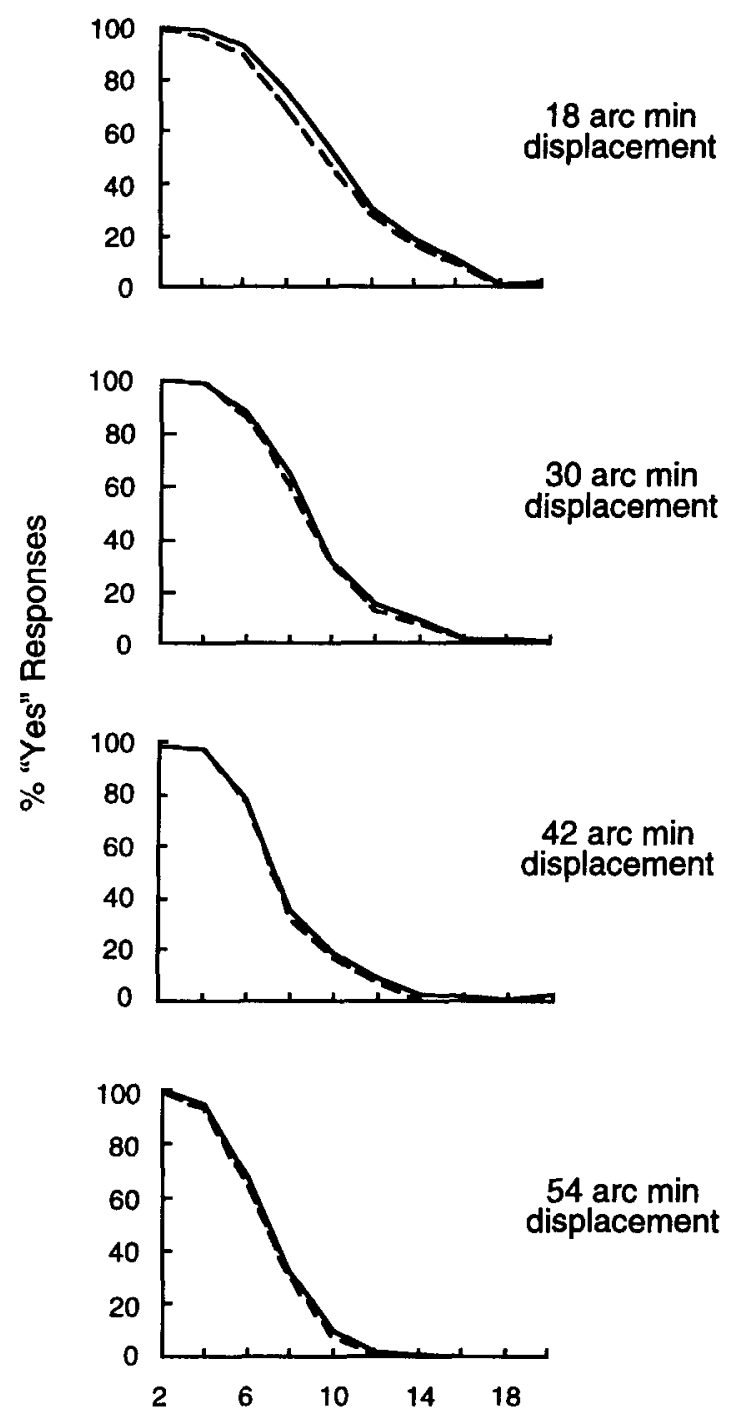

TEMPORAL ASYMMETRY
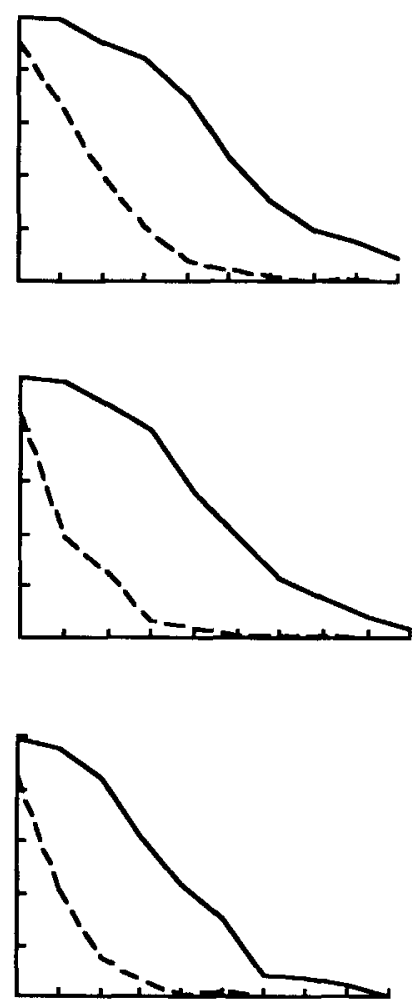

Number of Noise Dots

$$
\begin{gathered}
\text { Perception of } \\
\text { the Figure }
\end{gathered}
$$

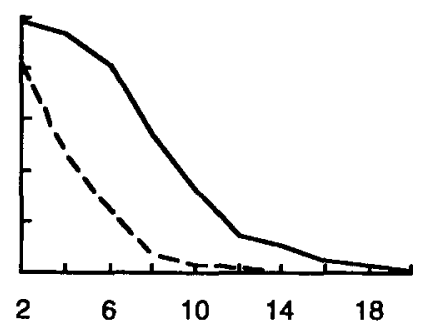

Figure 1. Experiment 1: The percentage of trials for which the subject responded yes as a function of noise level for the four figure displacements in the temporal symmetry and temporal asymmetry conditions. Psychometric functions (averaged over the 4 subjects) are shown for perceiving the figure (solid lines) and for perceiving the figure in motion (dashed lines). In the temporal symmetry condition, either a moving figure or random motion was perceived. In contrast, a moving figure, a stationary figure, or random motion was perceived in the temporal asymmetry condition.

of temporal symmetry, it always was perceived as moving. Figure stationarity emerged only under conditions of temporal asymmetry.

Perceptual switching. Perception near the $50 \%$ thresholds was bistable. In the temporal symmetry condition, figure motion and random motion were perceived equally often for the same noise values. They were perceptually distinct, with no intermediate state of uncertainty. In the temporal asymmetry condition, figure motion and figure stationarity were perceived equally often for the same noise value. They were again perceptually distinct, with no intermediate state of uncertainty.

When asked, at the conclusion of Experiment 1, the subjects reported few, if any, perceptual switches within 
TEMPORAL SYMMETRY

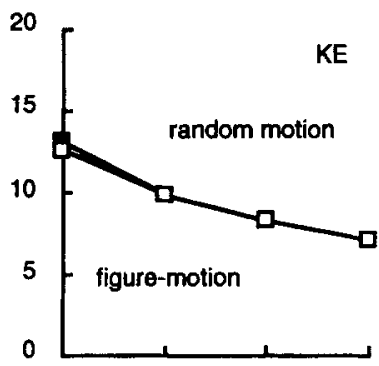

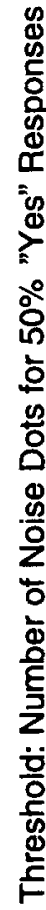

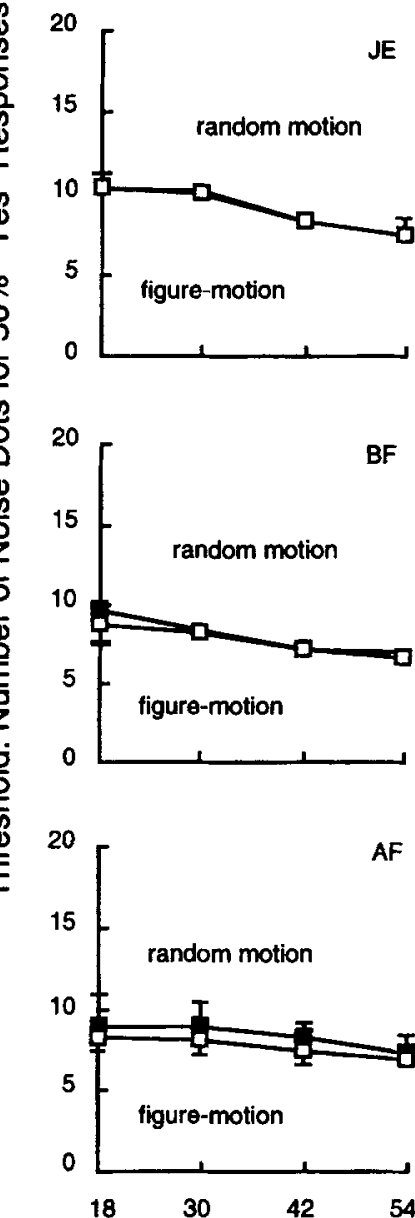

TEMPORAL ASYMMETRY
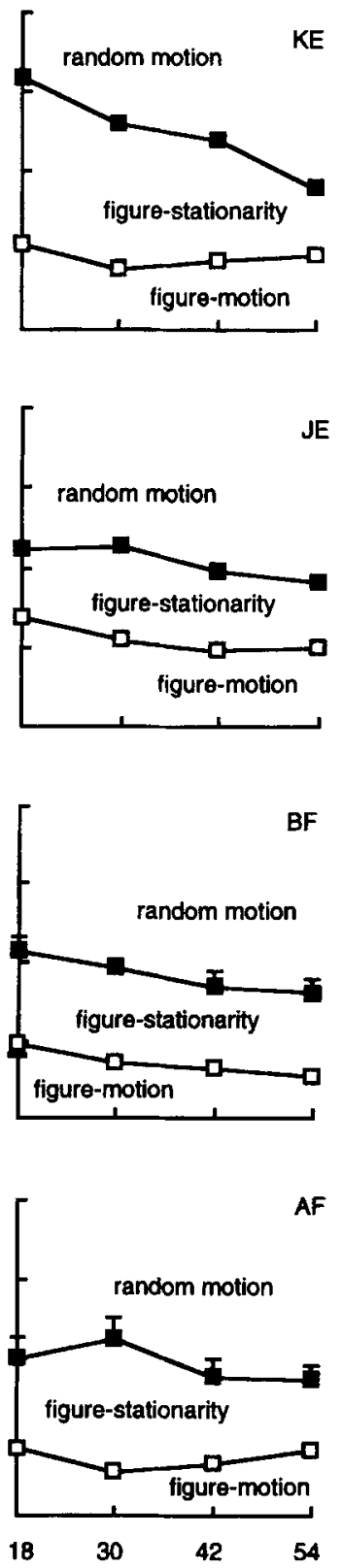

Displacement (in arc min)

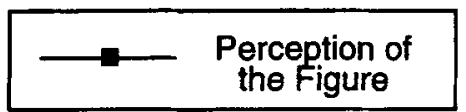

Perception of the Figure in Motion

Figure 2. Experiment 1: Each subject's probit-determined $50 \%$ thresholds (i.e., the number of noise dots resulting in yes responses on half the trials) for perceiving the figure (solid squares) and for perceiving the figure in motion (open squares) in the temporal symmetry and temporal asymmetry conditions. Standard errors (based on thresholds calculated for each of the eight testing sessions) are presented with each mean threshold, but only where they are larger than the symbol signifying the mean. 
individual trials - for example, a trial in which figure motion was initially perceived but then was spontaneously replaced by the perception of random motion. To confirm these reports, we conducted an additional experiment in which 2 of the 4 subjects tested in Experiment 1 (K.E. and J.E.) were asked to indicate after each trial whether or not a switch had occurred at any time during the trial. The subjects were tested in two 100-trial sessions in both the temporal asymmetry and the temporal symmetry conditions (in the 30 arc min displacement condition only). The mean switching rates were $0 \%$ (for K.E.) and $1.5 \%$ (for J.E.) in the temporal symmetry condition and $2.5 \%$ (for K.E.) and $2 \%$ (for J.E.) in the temporal asymmetry condition.

The absence of switching was a critical feature in concluding that the competing percepts were temporally stable. This followed from the calculation of what the switching rate would have been if every two-frame display cycle provided an independent opportunity for the occurrence of the competing percepts (Hock et al., 1997). If this were the case and if the occurrence of each percept was equally probable during each display cycle ( $p=.5$ at the $50 \%$ threshold), the probability of a switch sometime during the four-display-cycle trial was $\left(1-p^{3}\right)$, or .88 . Since the switching rates in both conditions were negligible, it could be concluded that the motion percept was temporally stable in its competition with random motion (in the temporal symmetry condition) and with stationarity (in the temporal asymmetry condition).

\section{EXPERIMENT 2}

It might be argued that the bistabilities observed in Experiment 1 were the fortuitious result of random changes in the locations of the noise dots. That is, since the location of the dots was changed randomly on every frame, it is possible that whether figure motion, figure stationarity, or random motion was perceived near the $50 \%$ thresholds depended on whether or not the noise dots happened to have been in locations that masked or unmasked the figure (in one or both of its lateral positions). Although the temporal stability of the percepts over each eight-frame trial argues strongly against this possibility, it can be completely excluded by evidence for perceptual bistability based on hysteresis effects. This was done at both the figure-motion/random-motion boundary and the figure-motion/figure-stationarity boundary.

Testing for hysteresis involves gradually increasing and decreasing a critical parameter in such a way that two distinct percepts are formed for the same parameter value, one predominating when the value is reached by a gradual increase in the parameter, the other when it is reached by a gradual decrease in the parameter. Hysteresis in the perception of coherent versus random motion for random cinematograms has previously been reported by Williams, Phillips, and Sekuler (1986); their manipulated parameter was the range of motion directions from which signal dots were drawn (as in the present study, the signal dots were embedded in a background of randomly moving dots).

In this experiment, the manipulated parameter was the number of noise dots; it was gradually increased or gradually decreased within each trial. Hysteresis was indicated by the continuous perception of the initially established percept, despite changes in the number of noise dots to values favoring the alternative percept. For example, the initial perception of figure stationarity could persist despite decreases in the number of noise dots to values favoring the perception of figure motion. As fortuitous noise-dot locations that mask or unmask the figure were equally likely for ascending and descending trials, they could not account for the bistability indicated by the hysteresis effects.

\section{Method}

Stimuli. The temporal symmetry and asymmetry conditions were the same as those in Experiment 1. The figure, which was again composed of three vertically aligned dots, was displaced back and forth by 30 min during alternating frames. Ascending trials (i.e., trials with a successively increasing number of noise dots) and descending trials (i.e., trials with a successively decreasing number of noise dots) were presented with noise levels appropriate for crossing the figure-motion/random-motion boundary in the temporal symmetry condition and with noise levels appropriate for crossing the figure-motion/figure-stationarity boundary in the temporal asymmetry condition. The selected noise values were based on the $50 \%$ thresholds measured for individual subjects in Experiment 1 ; one noise value was near the threshold, and three noise values were selected on either side of the boundary.

The experiment was based on the modified method-of-limits procedure devised by Hock, Kelso, and Schöner (1993). This procedure differs from the standard method of limits in two respects: (1) It eliminates potential confounding of perceptual hysteresis with response hysteresis by having subjects withhold their response until the end of each trial (rather than responding at each step in a gradually increasing or decreasing series); subjects indicate whether or not there had been a perceptual switch any time during the trial; and (2) each trial varies randomly with respect to how far the ascending or descending series penetrates into the transition zone (near the $50 \%$ threshold). The greater the penetration, the greater the likelihood of a perceptual switch.

For ascending trials, a noise level that resulted in the consistent perception of figure motion was repeated for the first three display cycles (a display cycle constituting the two-frame presentation of the figure in both its locations). Then, the number of noise dots was increased by one per display cycle, until an endpoint value was reached. Thus, a trial in the temporal symmetry condition with the sequence 7-7-7-8-9-10, which started with 7 and ended with 10 noise dots, penetrated further into the transition zone than did a trial with the sequence $7-7-7-8-9$, so a perceptual switch was more likely for the former. ${ }^{2}$ Descending trials were formed in the same way; noise levels sufficient for the consistent perception of random motion (in the temporal symmetry condition) or figure stationarity (in the temporal asymmetry condition) were selected for the initial three cycles, followed by a decreasing number of noise elements on each successive display cycle (e.g., 13-13-13-12-11-10-9).

Design. Ascending and descending noise levels, as well as trials with different endpoint noise values, were randomly mixed within one block of 80 trials in the temporal symmetry condition and within one block of 80 trials in the temporal asymmetry condition. Both blocks of trials were tested during each of three testing sessions (their order was balanced). 
Procedure. For the block of trials crossing the figure-motion/ random-motion boundary (the temporal symmetry condition), the subjects indicated, after each trial, whether they initially perceived the moving figure or just random motion, and then whether or not there was a switch to the alternative percept at any time later in the trial. For the block of trials crossing the figure-motion/figurestationarity boundary (the temporal asymmetry condition), the subjects indicated, after each trial, whether they initially perceived the figure as moving or stationary, and then whether there was a switch to the alternative percept at any time later in the trial.

Preliminary testing for each subject confirmed that the ranges of noise values tested for each condition were appropriate for the two response choices - that is, the subjects perceived only figure motion and random motion in the temporal symmetry condition and only figure motion and figure stationarity in the temporal asymmetry condition.

Subjects. Three of the 4 subjects from Experiment 1 participated in this experiment

\section{Results}

The percentages of descending trials (noise dots reduced on successive frames) for which random motion (Figure 3 ) and figure stationarity (Figure 4) were perceived without any switches to the perception of figure motion are graphed with respect to the axes on the left side of the graphs. The percentages of ascending trials (noise dots increased on successive frames) for which figure motion was perceived without any switches to the perception of random motion (Figure 3 ) or figure stationarity (Figure 4) are graphed with respect to the inverted axes on the right side of the graphs.

In the absence of hysteresis, the results for the descending and ascending trials would have overlapped. For example, initially perceived figure motion would have switched to the perception of figure stationarity at the same noise value as that at which initially perceived figure stationarity switched to the perception of figure motion. The results, however, provided evidence for hysteresis effects of similar magnitude at both the figure-motion/ random-motion boundary (Figure 3 ) and the figuremotion/figure-stationarity boundary (Figure 4). That is, perception strongly depended on the direction of parameter change. What was perceived for a particular noise value was strongly determined by what was perceived during earlier frames when the noise value was higher or lower, and as a result, the initially established percept persisted despite parameter changes to values favoring the competing percept. As was indicated in the introduction to this experiment, if perceptual bistability in Experiment 1 was the result of fortuitous locations of noise dots masking and unmasking the figure, there would not have been a difference between ascending and descending trials in this experiment.

\section{EXPERIMENT 3}

The results of Experiments 1 and 2 provided evidence for bistability with respect to whether a figure in motion or a single, stationary figure was perceived. The purpose of this experiment was to determine whether the stationary figure perceived in the temporal asymmetry condition

\section{Figure-Motion vs. Random-Motion}
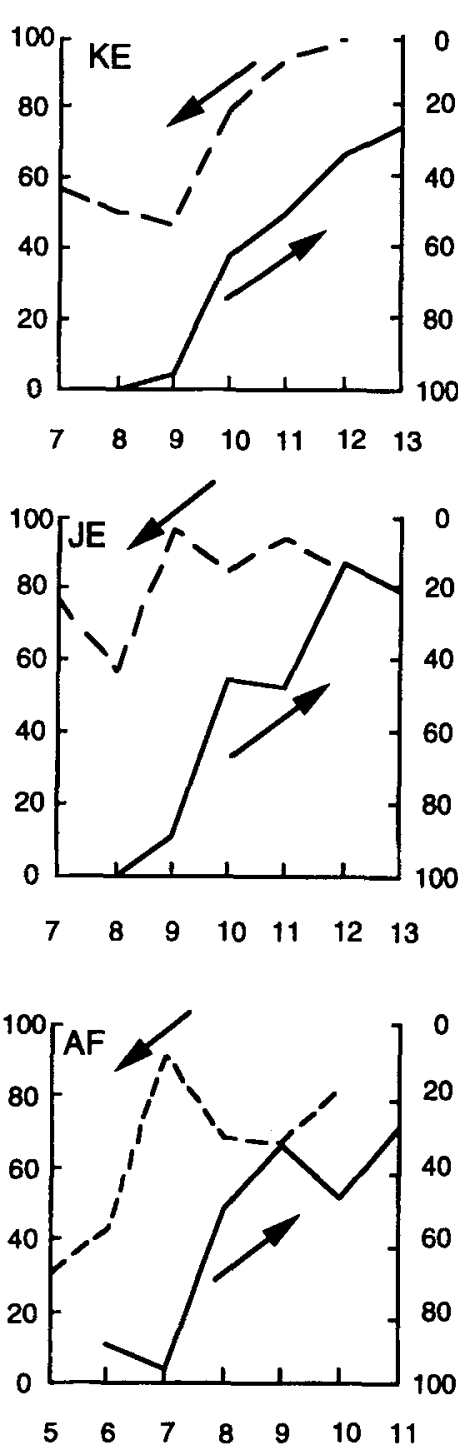

\section{Number of Noise Dots at the End of Each Trial}

Figure 3. Experiment 2, temporal symmetry condition: Hysteresis effects were assessed by gradually increasing or gradually decreasing the number of noise dots during each pair of frames (i.e., each display cycle) and by varying the number of noise dots presented during the final display cycle of each trial. The percentage of descending trials for which random motion was perceived without the percept switching to figure motion is indicated by the axes on the left side of the graphs. The percentage of ascending trials for which figure motion was perceived without the percept switching to random motion is indicated by the inverted axes on the right side of the graphs.

was continuously perceived as being in the same position. That is, was it always perceived where it was presented during the long-duration frame, or during the shortduration frame, or did its perceived position change (without motion perception) during the course of a trial? 
Figure-Motion vs. Figure-Stationarity

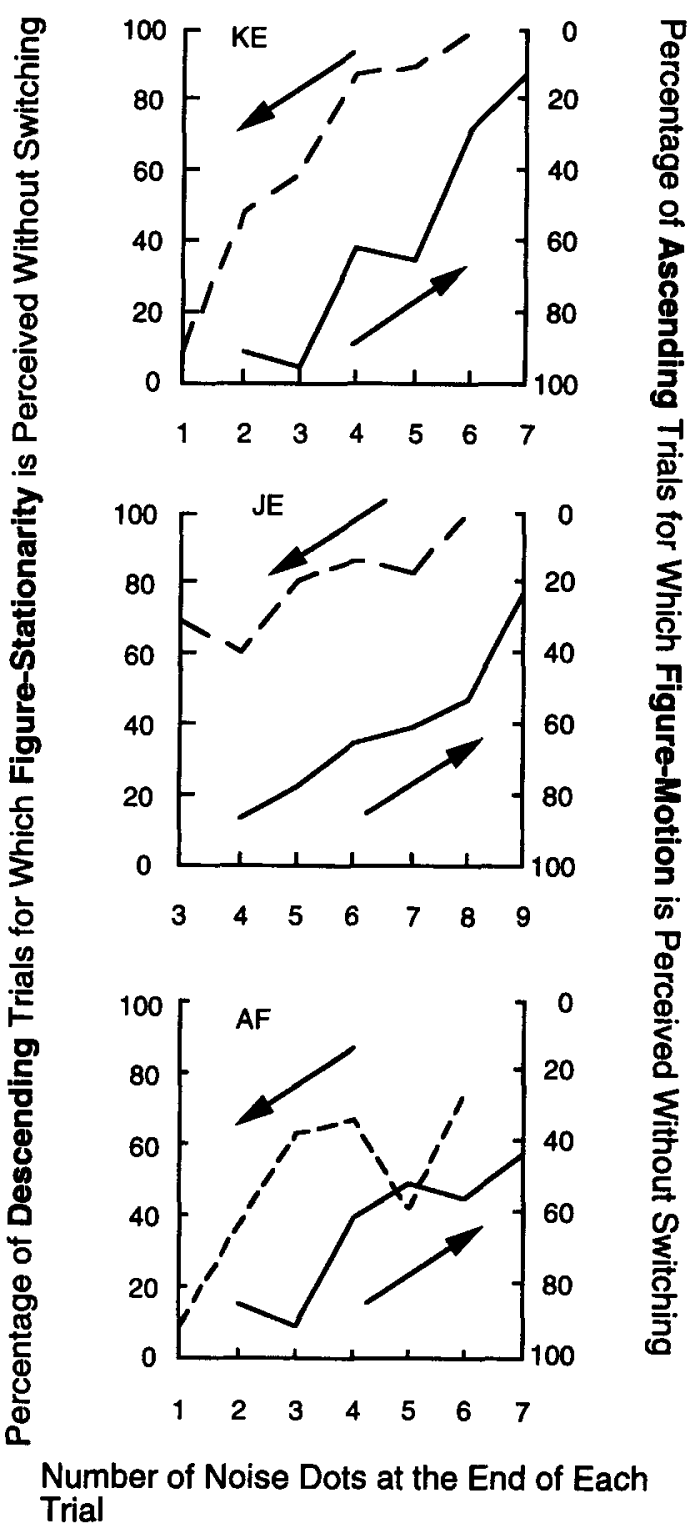

Figure 4. Experiment 2, temporal asymmetry condition: Hysteresis effects were assessed by gradually increasing or gradually decreasing the number of noise dots during each pair of frames (i.e., each display cycle) and by varying the number of noise dots presented during the final display cycle of each trial. The percentage of descending trials for which figure stationarity was perceived without the percept switching to figure motion is indicated by the axes on the left side of the graphs. The percentage of ascending trials for which figure motion was perceived without the percept switching to figure stationarity is indicated by the inverted axes on the right side of the graphs.

On the basis of the results of the previous experiment, noise levels were selected in the temporal asymmetry condition that strongly favored the perception of a single, stationary figure. The essential modification in this experiment was that the color of the three-dot figure was changed from white to red on the last frame of each trial (so that the hidden figure would pop-out of the noise, allowing motion to be perceived). For half the trials, the last frame was a long-duration frame; for the other half, it was a short-duration frame. If motion is perceived at the end of the trial when the color pop-out was on a trial-ending, short-duration frame, it would indicate that, until the color change, the figure had been perceived as being at the location it occupied during the long-duration frame (and vice versa, if motion was perceived at the end of the trial when the color pop-out was on a trial-ending, long-duration frame).

\section{Method}

The temporal asymmetry condition was the same as that in the preceding experiments (the temporal symmetry condition was not included). The three-dot figure was the same as those in the preceding experiments; its horizontal displacement was always 30 arc min. There was only one level of noise (eight randomly located dots), and the frame duration again alternated between 225 and $75 \mathrm{msec}$ on successive frames.

For half the trials, the figure was white until it changed to red during the 225-msec frame that concluded the trial; for the other half (randomly mixed), it was white until it changed to red during the $75-\mathrm{msec}$ frame that concluded the trial. The same 4 subjects as those in Experiment 1 participated in a single, 200-trial testing session. After each trial, the subjects first indicated whether the figure appeared to be stationary for the entire trial prior to the color change, and then whether the figure moved when its color changed from white to red at the end of the trial.

\section{Results}

The percentages of trials for which figure motion was perceived when the figure changed color during trialending, long-duration frames or during trial-ending, shortduration frames are presented in Table 1. Only the results of trials for which a stationary (white) figure was perceived for the entire trial preceding the color change were analyzed (very few trials were excluded).

It was found that the figure did not appear to move when it changed from white to red during a trial-ending, long-duration frame. This indicated that, prior to the color pop-out, the stationary figure already was being perceived at the location in which it had been presented during the long-duration frame. When the color pop-out occurred during a trial-ending, short-duration frame, motion was almost always perceived. That is, when the white figure changed to red, it was perceived as moving to the location in which it had previously not been perceived. This provided further evidence that, prior to the color change, the stationary figure was only perceived in the figure's location during the long-duration frame.

Table 1

Experiment 3: Percentage of Trials for Which Motion is Perceived When the White Figure Changes to Red During the Last Frame

\begin{tabular}{ccc}
\hline & \multicolumn{2}{c}{ Duration of Trial-Ending Frame } \\
\cline { 2 - 3 } Subject & $75 \mathrm{msec}$ & $225 \mathrm{msec}$ \\
\hline K.E. & 100 & 3 \\
J.E. & 94 & 8 \\
B.F. & 94 & 0 \\
A.F. & 85 & 0 \\
\hline
\end{tabular}


It could be concluded, therefore, that when the displaced figure was perceived as being stationary in the temporal asymmetry condition, it was always perceived as being in a single position, the position corresponding to its location during the long-duration frame. If the figure had been consistently perceived as being stationary in a position between its alternating locations or if the perceived position of the figure changed during the trial (without motion perception), the figure would have appeared to move when the color pop-out occurred on trialending, long-duration, as well as on trial-ending, shortduration frames.

\section{GENERAL DISCUSSION}

The results of the three experiments reported in this article provided evidence for bistability with respect to whether a figure was perceived as being in motion or a single, stationary figure was perceived in a fixed position (the temporal asymmetry condition) and for bistability with respect to whether a figure in motion or random dot motion was perceived (the temporal symmetry condition). The bistability near the $50 \%$ threshold in the temporal asymmetry condition was consistent with Hock et al.'s (1996), and Hock et al.'s (1997) results. However, in the present case, the perception of stationarity, or nonmotion, could be more directly linked with the perception of the figure's position (otherwise, the stationary figure could not have been perceived against the background of noise dots).

The bistability in the temporal asymmetry condition reflects the stability or state dependence of the figuremotion and figure-stationary percepts near the threshold; what was perceived was highly influenced by the immediately preceding perceptual state. As in Hock et al. (1997), it could be concluded that perception near the $50 \%$ threshold was dynamic. That is, in contrast with the classical conception, in which the $50 \%$ threshold reflects a state of uncertainty regarding which of the alternatives is being perceived, a dynamic threshold is one in which the alternatives are perceived equally often, but each is perceptually distinct, with relatively little switching between them.

The observed bistability reflects the presence of inhibitory competition between motion and position perception. The existence of strong inhibition is necessary in order to account for the suppression of motion perception when a single, stationary figure was perceived, and vice versa. Expressed neurophysiologically (Orban et al., 1986), what is perceived depends on whether the ensemble of bandpass cortical neurons (motion detectors) or the ensemble of lowpass cortical neurons (position detectors) is more strongly activated, with inhibitory interactions further reducing the activity of the more weakly activated ensemble. Motion of the horizontally displaced figure is perceived when the activation of bandpass neurons selective to horizontal motion sufficiently exceeds the activation, produced by the randomly moving noise dots, of detectors selective to other motion directions.

Why, then, was figure stationarity perceived in the temporal asymmetry, but not in the temporal symmetry condition? It cannot be attributed to perceptual persistence (iconic memory) spanning the brief, 75-msec interval between the 225 -msec frames; figure stationarity is never perceived when the frame duration is $75 \mathrm{msec}$ for both figure locations. A more likely reason for the emergence of the stationarity percept in the temporal asymmetry condition is based on our evidence for motion/ stationarity bistability, which indicates the presence of competition between the perception of motion and that of position. This competition suggests that either the activation of the motion-detecting ensemble must be decreased or the activation of the position-detecting ensemble must be increased, in order for the stationary figure to be perceived without motion perception (and then only in the location where it is presented for a relatively long time).

These alternatives were evaluated through the Fourier transformation of the time-varying luminance at each figure location (based on the eight frames constituting each trial). The resulting temporal frequency spectra (Figure 5) indicate the amplitude (in arbitrary units) of a continuous series of component frequencies so that the original stimulus can be reconstructed by the summation (integration) of these temporal frequency components. It can be seen in Figure 5 that, in comparison with the temporal symmetry condition, the maximum amplitude of time-varying energy (the fundamental) is decreased at the long- and brief-duration figure locations in the temporal asymmetry condition but increased at higher temporal frequencies (the second harmonic). Given that all these frequency components can potentially contribute to the perception of motion (Schrater, 1998; van Santen \& Sperling, 1985), there was no clear difference in motion energy between the temporal symmetry and the temporal asymmetry conditions. However, it can also be seen in Figure 5 that, in comparison with the temporal symmetry condition, the $0-\mathrm{Hz}$ (time-averaged) energy in the temporal asymmetry condition is substantially increased at the long-duration location of the figure (consistent with the increased activation of position detectors and, thus, the increased likelihood of seeing a stationary figure at this location) and substantially decreased at the brief-duration location of the figure (consistent with decreased activation of position detectors and, thus, the failure to see a stationary figure at this location). It thus appears to be the asymmetry in $0-\mathrm{Hz}$ energy that is the likely reason for the emergence of the stationary figure percept for temporally asymmetric presentations.

Finally, the results of this study provide a basis for evaluating other phenomena for which mutual inhibition between motion and position detectors could be the basis for perceptual bistability. The most notable of these is the bistability observed for the Ternus configuration (Ter- 

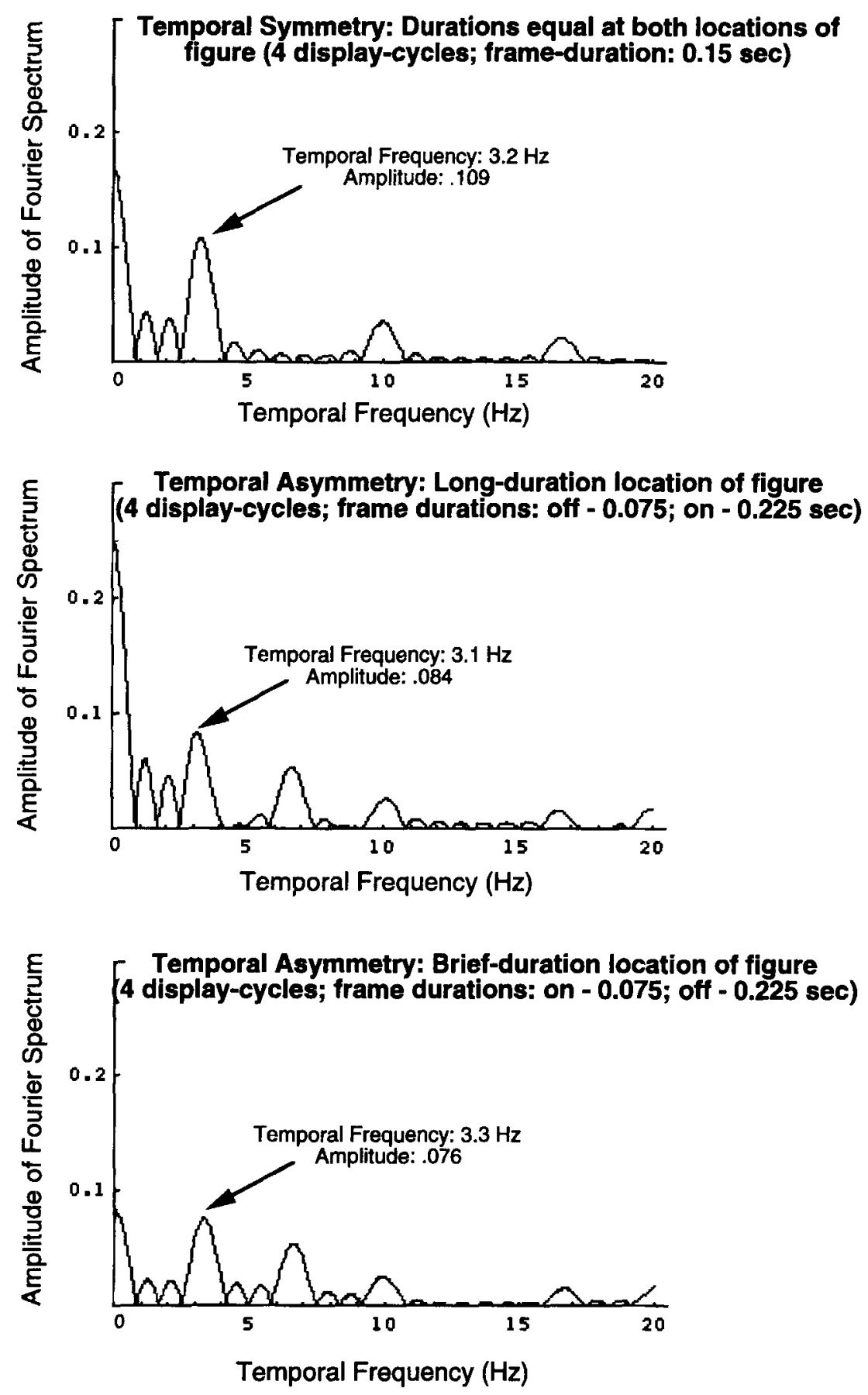

Figure 5. Fourier transforms based on the time-varying luminance at each of the figure locations in the temporal symmetry and temporal asymmetry conditions.

nus, 1926), for which a short row of equally spaced dots is horizontally displaced on successive frames by a distance equal to the interdot spacing. Pantle and Picciano (1976) created bistability with the Ternus configuration by introducing a blank IFI between successive frames. They found that motion was perceived only between the "outside" dot locations, which were occupied by dots during alternating frames (the "inside" dot locations, which were occupied on every frame, were perceived as stationary), or group motion was perceived for the entire row of dots (including the "inside" dot locations). Early accounts of this bistability attributed it to competition between short- and long-range motion processes (Braddick, 1980; Pantle \& Picciano, 1976). More recently, Grossberg and Rudd (1992) have modeled the Ternus bistability through interactions between transient and sustained 
cells. Our evidence that motion and position detection can compete provides psychophysical evidence for the plausibility of Grossberg and Rudd's model. Most important, our results show that the failure to perceive motion does not necessarily mean that motion detectors are inadequately stimulated. What matters for motion perception is whether they are stimulated more than competing position detectors.

\section{REFERENCES}

BRADDICK, O. J. (1974). A short-range process in apparent motion. $\mathrm{Vi}$ sion Research, 14, 519-527.

BRADDICK, O. J. (1980). Low-level and high-level processes in apparent motion. Philosophical Transactions of the Royal Society of London: Series $B, 290,137-151$.

CAsCo, C., \& Morgan, M. (1987). Detection of moving local density differences in dynamic random patterns by human observers. Perception, 16, 711-717.

Casco, C., Morgan, M., \& Ward, M. (1989). Spatial properties of mechanisms for detection of moving targets in dynamic visual noise. Perception, 18, 285-291.

CavanaGh, P. (1992). Attention-based motion perception. Science, 257, 1563-1565.

Grossberg, S., \& RudD, M. E. (1992). Cortical dynamics of visual motion perception: Short-range and long-range apparent motion. $P_{s y c h o-}$ logical Research, 99, 78-121.

Hock, H. S., Balz, G. B., \& Eastman, K. E. (1996). Cooperative interactions and the perception of motion and stationarity for directionally ambiguous apparent-motion stimuli. Perception, 25, 887-900.

Hock, H. S., Kelso, J. A. S., \& Schöner, G. (1993). Bistability and hysteresis in the organization of apparent motion patterns. Journal of Experimental Psychology: Human Perception \& Performance, 19, 63-80.

Hock, H. S., Kogan, K., \& Espinoza, J. K. (1997). Dynamic, statedependent thresholds for the perception of single-element apparent motion: Bistability from local cooperativity. Perception \& Psychophysics, 59, 1077-1088.

ORBAN, G. A., KENNEDY, H., \& BUllier, J. (1986). Velocity sensitivity and direction selectivity of neurons in Areas V1 and V2 of the monkey: Influence of eccentricity. Journal of Neurophysiology, 56, 462-480.

PANTle, A. J., \& Picciano, L. (1976). A multistable movement display: Evidence for two separate motion systems in human vision. Science, 193, 500-502.

POSNER, M. I. (1980). Orienting of attention. Quarterly Journal of Experimental Psychology, 32, 3-25.

Ramachandran, V. S., \& ANSTis, S. M. (1983). Displacement thresholds for coherent apparent motion in random dot-patterns. Vision Research, 23, 1719-1724.

SCHRATER, P. R. (1998). Evidence for local velocity detectors: Additivity and selective pooling of spatio-temporal frequencies consistent with local translation [Abstract]. Investigative Ophthalmology \& Visual Science, 39, S426.

TERNUS, J. (1926). Experimentelle Untersuchungen über phänomenale Identät [Experimental investigations of phenomenal identity]. Psycologische Forschung, 7, 81-136.

van Santen, J. P., \& SPERLing, H. G. (1985). Elaborated Reichardt detectors. Journal of the Optical Society of America A, 2, 300-320.

Williams, D., Phillips, G., \& Sekuler, R. (1986). Hysteresis in the perception of motion direction as evidence for neural cooperativity. Nature, 324, 253-255.

\section{NOTES}

1. Accurate timing of the software used to generate visual stimuli was verified with a Tektonix TDS 544A four-channel digitizing oscilloscope.

2. Although trials with different endpoint values differed in total duration, previous studies (Hock et al., 1993; Hock et al., 1997) have shown that hysteresis effects continue to be obtained when total trial duration is controlled.

(Manuscript received September 15, 1997; revision accepted for publication June 23,1998 .) 